\title{
Numerical Investigation into the Effect of Splats and Pores on the Thermal Fracture of Air Plasma-Sprayed Thermal Barrier Coatings
}

\author{
Jayaprakash Krishnasamy $^{1} \cdot$ Sathiskumar A. Ponnusami $^{2} \cdot$ Sergio Turteltaub $^{1} \cdot$ \\ Sybrand van der Zwaag ${ }^{1}$
}

Submitted: 1 July 2019/in revised form: 24 October 2019/Published online: 13 November 2019

(C) The Author(s) 2019

\begin{abstract}
The effect of splat interfaces on the fracture behavior of air plasma-sprayed thermal barrier coatings (APS-TBC) is analyzed using finite element modeling involving cohesive elements. A multiscale approach is adopted in which the explicitly resolved top coat microstructural features are embedded in a larger domain. Within the computational cell, splat interfaces are modeled as being located on a sinusoidal interface in combination with a random distribution of pores. Parametric studies are conducted for different splat interface waviness, spacing, pore volume fraction and fracture properties of the splat interface. The results are quantified in terms of crack nucleation temperature and total microcrack length. It is found that the amount of cracking in TBCs actually decreases with increased porosity up to a critical volume fraction. In contrast, the presence of splats is always detrimental to the TBC performance. This detrimental effect is reduced for the splat interfaces with high waviness and spacing compared to those with low waviness and spacing. The crack initiation temperature was found to be
\end{abstract}

Jayaprakash Krishnasamy

J.Krishnasamy-1@tudelft.nl

Sathiskumar A. Ponnusami

sathiskumar.ponnusami@city.ac.uk

Sergio Turteltaub

S.R.Turteltaub@tudelft.nl

Sybrand van der Zwaag

S.vanderZwaag@tudelft.nl

1 Faculty of Aerospace Engineering, Delft University of Technology, Kluyverweg 1, 2629 HS Delft, The Netherlands

2 Department of Mechanical Engineering and Aeronautics, City, University of London, Northampton Square, London EC1V 0HB, UK linearly dependent on the normal fracture properties of the splat interface. Insights derived from the numerical results aid in engineering the microstructure of practical TBC systems for improved resistance against thermal fracture.

Keywords cohesive elements $\cdot$ fracture $\cdot$ porosity $\cdot$ splats $\cdot$ thermal barrier coatings

\section{Introduction}

Thermal barrier coatings (TBC) are heat insulation layers applied on the high-temperature regions of aircraft jet engines in order to protect the crucial structural components against overheating and consequently to extend the lifetime of these components. They also improve the combustion efficiency of the engine by allowing higher operational temperature. The most common TBC system is a multilayered system. It consists of two layers; an outer ceramic layer that protects the substrate from high-temperature gases and an intermediate metallic layer which provides adhesion between the substrate and the ceramic layer. The metallic layer also protects the substrate from high-temperature oxidation and corrosion. The outer ceramic layer is called top coat (TC) and is often made of yttria stabilized zirconia (YSZ). The metallic layer is called bond coat (BC) and is usually made of MCrAlY (M refers to Ni or $\mathrm{Al}$ or a mixture of these two. The coating system is subjected to a thermal cycle during operation where the temperature ranges from ambient to its operating value (around $1200^{\circ} \mathrm{C}$ ). During operation, a thin thermally grown oxide (TGO) layer consisting of alumina $\left(\mathrm{Al}_{2} \mathrm{O}_{3}\right)$ is formed at the TC/BC interface due to oxidation of the bond coat. During each thermal cycle, thermal stresses are generated in the coating due to a mismatch in coefficients of thermal 
expansion (CTE) of the TBC layers. These thermal stresses along with the growth of the TGO layer lead to the nucleation and evolution of microcracks in the coating as illustrated in Fig. 1. With the increase in thermal cycles, these microcracks coalesce to form a larger crack which results in final failure of the coating known as spallation.

Conventional TBCs are predominantly manufactured either by an electron beam physical vapor deposition (EBPVD) process or the air plasma spray (APS) technique (Ref 1). In the EB-PVD technique, the coatings are deposited on the substrate in vacuum using a high-energy electron beam (Ref 2). This technique produces a uniform coating with a fine columnar grain structure. These coatings offer superior strain tolerance, thermal shock and oxidation resistance which ultimately leads to prolonged service life. In the APS method, ceramic particles are melted and sprayed onto the substrate at atmospheric pressure using plasma jet (Ref 3). APS TBCs have a shorter lifetime compared to EBPVD TBCs due to its layered microstructure and higher number of defects. However, APS remains the preferred manufacturing method in the fields of aerospace and power generation gas turbines because of its low cost and high production efficiency (Ref 4). A schematic diagram of the microstructure of the APS TBC and its failure behavior is presented in Fig. 1.

The distinctive microstructural features of APS TBCs are splats in addition to other features such as pores, microcracks and interface roughness. Splats are building blocks of the APS TBCs which are formed during the solidification process of the sprayed ceramic particles (Ref 5). The interfaces between the splats are usually weak. The characteristics of the splat interfaces are determined by the spraying parameters (Ref 6-8). Literature studies on TBC microstructure show that the presence of splats along with microstructural pores significantly influences the material properties and failure behavior of the TBC (Ref 9-13).

Multiple efforts have also been undertaken in the literature to model and predict the influence of splat interfaces on thermo-elastic properties of TBCs (Ref 14-17). In the work (Ref 14), real microstructural images are used to predict the effective thermal conductivity and stiffness using finite element analysis. The stiffness reduction due to splats is obtained indirectly by calculating the difference between the stiffness values of finite element prediction of as-sprayed (with splats) and experimental measurements of thermal-cycled (no splats) samples, respectively. Similar type of work is also carried out in (Ref 15) using imagebased extended finite element modeling. In addition, the evolution of the stress intensity factor and the quenching stress as a function of intra-splat cracks and substrate temperature is also analyzed in their study. Thermal conductivity of splat interfaces is obtained in (Ref 16) through iterative finite element computations and by mapping the calculated effective thermal conductivity with the experimental measurements. A finite element model is generated using real splat interface distribution obtained from fracture image analysis. A finite element analysis on idealized YSZ model system to analyze the effect of splat interfaces on thermal conductivity of TBC is carried out in (Ref 17).

Literature studies to predict the influence of all aspects of the TBC microstructure on its failure behavior are limited. Some studies are focussed only on the effect on TC/
Fig. 1 A schematic APS TBC system showing the failure mechanisms and the governing loading conditions
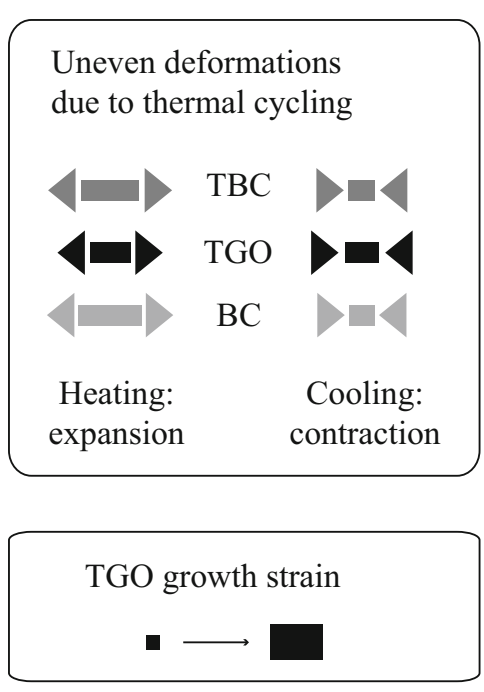

TBC loading conditions

(a)
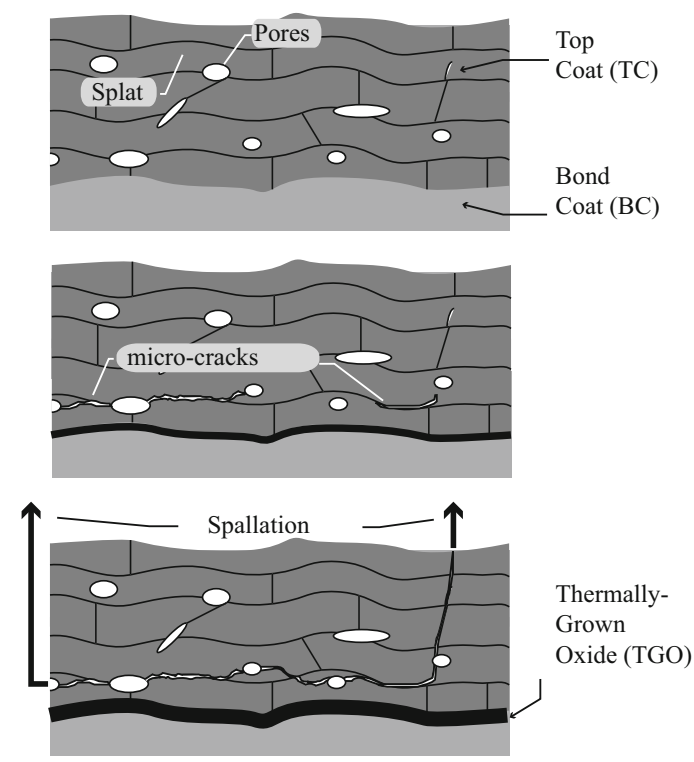

Crack coalescence and TBC failure

(b) 
BC interface and microstructural pores (Ref 18, 19). A recent numerical study has also included the presence of splats, although authors have considered a single type of microstructure (Ref 20). Experimental observations show that the failure behavior of the TBC is significantly influenced by the splats (Ref 11, 21, 22). For instance, in addition to the microstructural pores, the stacking configuration and the bonding between the splats are also important for the fracture resistance of coatings (Ref 21). It is, therefore, the objective of this research to carry out a systematic numerical study to investigate the effect of interface topology and its fracture strength on the TBC fracture behavior in the presence of microstructural pores. Furthermore, concurrent multiscale modeling setup is adopted to include the free edge effect which is relevant in terms of interpreting experimental results of coated samples. The effect of splat interfaces is investigated using a parametric $\mathrm{TBC}$ model with realistic pore distribution corresponding to various splat interface features, namely waviness, spacing and also the strength. The scope of this research is relevant to the TBC community for microstructure optimization to enable them to choose the right set of processing and spraying parameters to get the desired splat interface characteristics.

\section{Multiscale Finite Element Model of TBC Microstructure}

\section{Overall Geometry}

The modeling of the TBC is carried out in a two-dimensional domain under plane strain assumptions. A two-scale concurrent multiscale modeling approach is adopted in which the explicitly resolved TC microstructural features are embedded in a much larger domain of the TBC with an homogenized TC layer, as shown in Fig. 2. TBC microstructural features such as pores, interface roughness and splat interfaces are modeled explicitly in the TC layer of the embedded computational cell as depicted in Fig. 2(b).

The dimensions of the TBC geometry considered in this work are derived from the disk-shaped APS TBC samples developed within the research consortium (Ref 23) for microstructure characterization and thermal cycling behavior. The total thickness and the radius of the diskshaped sample are $6 \mathrm{~mm}$ and $15 \mathrm{~mm}$, respectively. The width of the embedded computational cell is considered as $480 \mu \mathrm{m}$ (Ref 24). More details about the geometry and the modeling setup can be found in (Ref 18).

TBC computational cell is enriched with pore features that are representative of TC porosity measurements obtained as part of the collaborative work (Ref 23). For conciseness, only the statistical distribution of the porosity measurements is provided in Table 1. The pores are modeled as an ellipsoidal quantity with an aspect ratio (AR), orientation and size given in Table 1 for modeling convenience. The pores are distributed randomly within the TBC computational cell.

For simplicity, the interface waviness between TC and $\mathrm{BC}$ and also the horizontal splat interfaces are represented as a sinusoidal curve. The vertical coordinate $y_{n}$ of the n-th splat interface is given by as a function of the horizontal coordinates $x$ as

$$
y_{n}(x)=A_{0} C^{n} \cos (x 2 \pi / \lambda)+n h
$$

where $A_{0}$ and $\lambda$ corresponds to the reference amplitude and wavelength of the sinusoidal curve, respectively. The subscript $n$ refers to the types of interfaces in the TC layer ( $n=0$ corresponds to the TC/BC interface and $n>0$
Fig. 2 A schematic of concurrent multiscale TBC model with detailed microstructure (a)

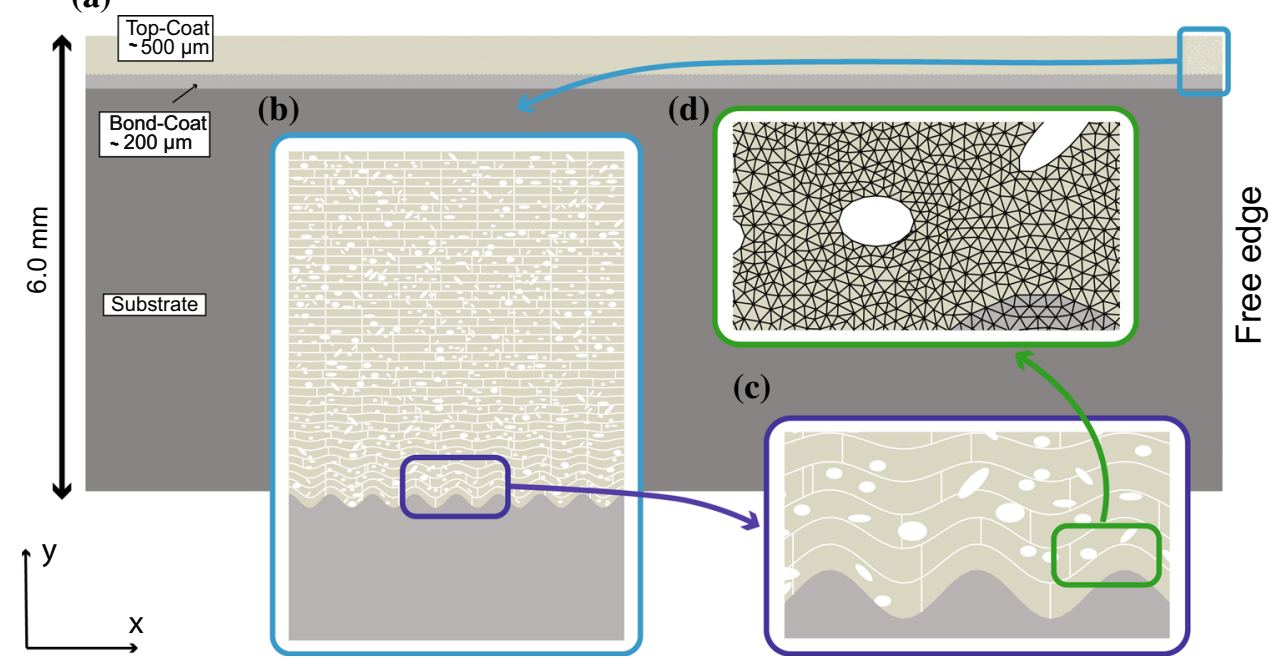


Table 1 Modeling parameters for random pore generation

\begin{tabular}{ll}
\hline Geometrical features & \multicolumn{1}{c}{ Modeling parameters, \% } \\
\hline Volume fraction & 10 \\
Micro-porosity (SP) & $5\left(\mathrm{size}=25 \mu \mathrm{m}^{2}\right)$ \\
Macro-porosity (LP) & $5\left(\mathrm{size}=75 \mu \mathrm{m}^{2}\right)$ \\
Round-shaped porosity & $5(\mathrm{AR}=1.5)[4 \mathrm{LP}, 4 \mathrm{SP}]$ \\
Lamellar-shaped porosity & $5(\mathrm{AR}=3)$ \\
Horizontally oriented lamellar porosity & $2.5\left(0^{\circ}\right)[1.75 \mathrm{LP}, 1.75 \mathrm{SP}]$ \\
Inclined lamellar porosity & $2.5\left( \pm 45^{\circ}\right)[1.75 \mathrm{LP}, 1.75 \mathrm{SP}]$ \\
\hline
\end{tabular}

corresponds to the splat interfaces), $h$ and $C$ denote the spacing between the two adjacent interface and interface waviness, respectively. The waviness parameter $C$ ranges from $C=0$ (for a flat interface) to $C=1$ (for a sinusoidal interface with amplitude $A_{0}$. The wavelength $(\lambda)$ and initial amplitude $\left(A_{0}\right)$ of the interface are assumed to be constant with a specified value of $60 \mu \mathrm{m}$ and $10 \mu \mathrm{m}$, respectively (Ref 24). The vertical splat interfaces are modeled as straight lines, and they are distributed randomly between the horizontal splat interfaces as shown in Fig. 2(c). The number of vertical splat interfaces between any two successive horizontal interfaces is set to a value of 10 over the length of the explicitly modeled domain.

\section{Simulation Setup}

The microstructure is meshed with three-noded plane strain triangular elements (CPE3) using GMSH to model the bulk response of the individual TBC layers. The fracture behavior of the TBC is modeled using a cohesive zone model which is implemented in the finite element framework using the zero thickness four-noded cohesive element (COH2D4). It is also important to mention that the fracture process is modeled only in the computational cell and the outer regions of the TBC only can respond as a homogenized featureless elastic medium. Also, the elastic response of the splat interfaces themselves are not considered in the analysis since the interfaces are very thin compared to the thickness of the splats. In other words, the constitutive behavior of the splat interfaces is governed only by its fracture properties, defined through zero thickness cohesive element. To enable arbitrary crack initiation and propagation, the cohesive elements are inserted at every bulk element interface of the TBC computational cell using a MATLAB script (Ref 25). However, the cracks are allowed to propagate only along inter-element boundaries which lead to a mesh dependency effect. Hence, to obtain a converged fracture pattern, a fine mesh size of $1 \mu \mathrm{m}$ is used in the computational cell region with a random mesh pattern that mitigates mesh dependency and provides meanvalue convergence. The outer elastic regions of the TBC are coarsely meshed with an average element size of 150 $\mu \mathrm{m}$ to reduce the computational time. The loading condition considered in this work corresponds to a single thermal cycle of as-deposited TBC system. A typical thermal cycle of a TBC consists of three phases namely the heating, dwell and cooling phase. In the heating and dwell phases of the thermal cycle, the TBC is assumed to be stress free as these coatings are deposited at high temperature. Hence, only the cooling phase of the thermal cycle is considered in the analysis, where the temperature is decreased from its typical operating value of 1100 to $30{ }^{\circ} \mathrm{C}$. Simulations are carried out under uniform temperature distribution which corresponds to the testing of samples in an oven (uniform temperature). The case of non-uniform temperature distributions has been studied experimentally in (Ref 26). Stresses appear in the TBC system during cooling due to the thermal contraction and the mismatch in coefficients of thermal expansion as shown in Fig. 1. The cooling rate does not play a role as the simulations are based on a quasistatic analysis. The nonlinear fracture simulations are carried out in the FEA package Abaqus using an implicit Newton-Raphson solver.

\section{Constitutive Models and Material Properties}

The bulk response of the different layers of TBC is assumed to be linearly elastic and isotropic. The fracture behavior of the TBC is modeled using a bilinear cohesive law (Ref 27) which is governed by the fracture strength and fracture energy. Material parameters used for individual TBC layers are summarized in Table 2. Elastic and thermal properties of the TBC layers are considered to be the same values used in the earlier work (Ref 18). The fracture properties such as normal strength and toughness of the TC, TGO and BC layers are in correspondence with the values reported in (Ref 28-30). The mode I fracture energies $\left(G_{\mathrm{IC}}\right)$ given in Table 2 are calculated from the toughness values $\left(K_{\mathrm{IC}}\right)$ under plane strain assumption. The ratio of shear strength to normal strength $(\gamma)$ for the TC layer, TGO layer and splat interfaces is assumed arbitrarily to have a value of 4 to suppress the failure of TBC in planes that are not parallel to the top surface in accordance with experimental observations. The mode I fracture 
Table 2 Elastic and fracture material parameters of the TBC components

\begin{tabular}{lcccccc}
\hline Layers & $E, \mathrm{GPa}$ & $v$ & $\alpha, 10^{-6} 1{ }^{\circ} \mathrm{C}$ & $\sigma_{n}, \mathrm{MPa}$ & $G_{\mathrm{IC}}, \mathrm{N} / \mathrm{mm}$ & $\gamma$ \\
\hline Top coat & 200 & 0.15 & 12.5 & 200 & 0.01 & 4 \\
Bond coat & 130 & 0.3 & 14.5 & 500 & 0.3 & 1 \\
Splat interface & $\ldots$ & $\cdots$ & $\cdots$ & 75 & 0.002 & 4 \\
TGO & 380 & 0.15 & 6 & 380 & 0.06 & 4 \\
Substrate & 200 & 0.28 & $\cdots$ & $\cdots$ & $\cdots$ & $\cdots$ \\
\hline
\end{tabular}

features given in Table 1. As discussed in the modeling setup, the horizontal splat interfaces are modeled using Eq 1 with the splat interface waviness and spacing of 0.8 and $12.5 \mu \mathrm{m}$, respectively. To include the effect of randomness of the pore distribution and vertical splat interfaces in the analysis, five different realizations are considered. To illustrate the effect of splat interfaces in the TBC failure process, stress distributions $\left(\sigma_{y y}\right)$ along with the crack pattern at the end of the thermal loading step for two different microstructural configurations (namely TC layer with pores and combined splat interfaces and pores) are shown in Fig. 3. The overall stress distribution $\left(\sigma_{y y}\right)$ is in correspondence with the studies carried out in the literature (Ref 19, 31). As observed in Fig. 3, the peaks and valleys of the $\mathrm{TC} / \mathrm{BC}$ interface lead to tensile and compressive states of stress $\left(\sigma_{y y}\right)$, respectively. The CTE mismatch between the layers and the presence of pores result in a complex stress field especially at the vicinity of pores as shown in Fig. 3. Also, for both configurations, the cracks tend to nucleate and propagate from the edge due to tensile stress $\left(\sigma_{y y}\right)$ generated out of the free edge accompanied by local stress concentration near the pores. However, as shown in Fig. 3(a), (b), the presence of splat interfaces has a significant effect on the TBC cracking behavior both in terms of crack nucleation and propagation. For the TBC with combined splat interfaces and pores, the cracks propagate mostly along the splat interfaces. In case of TBC with only pores, the crack propagation is governed by the direction of local stress concentration generated by the pores. It can also be observed that the effect of the cracking remains relatively local since the stress distribution in the TC sufficiently far away from the cracked regions remains identical for both configurations.

The results are presented in terms of crack initiation temperature and total crack length in Table 3 for the four distinct TBC microstructural configurations as discussed.

It can be observed from Table 3 that the variations in TBC microstructural configuration have a significant influence on its failure behavior. For the microstructural configurations without splat interfaces, the presence of pores did not have a substantial effect on crack initiation temperature compared to the dense case whereas a positive influence of pores is observed for the configurations with splat interfaces (i.e., a lower crack initiation temperature 


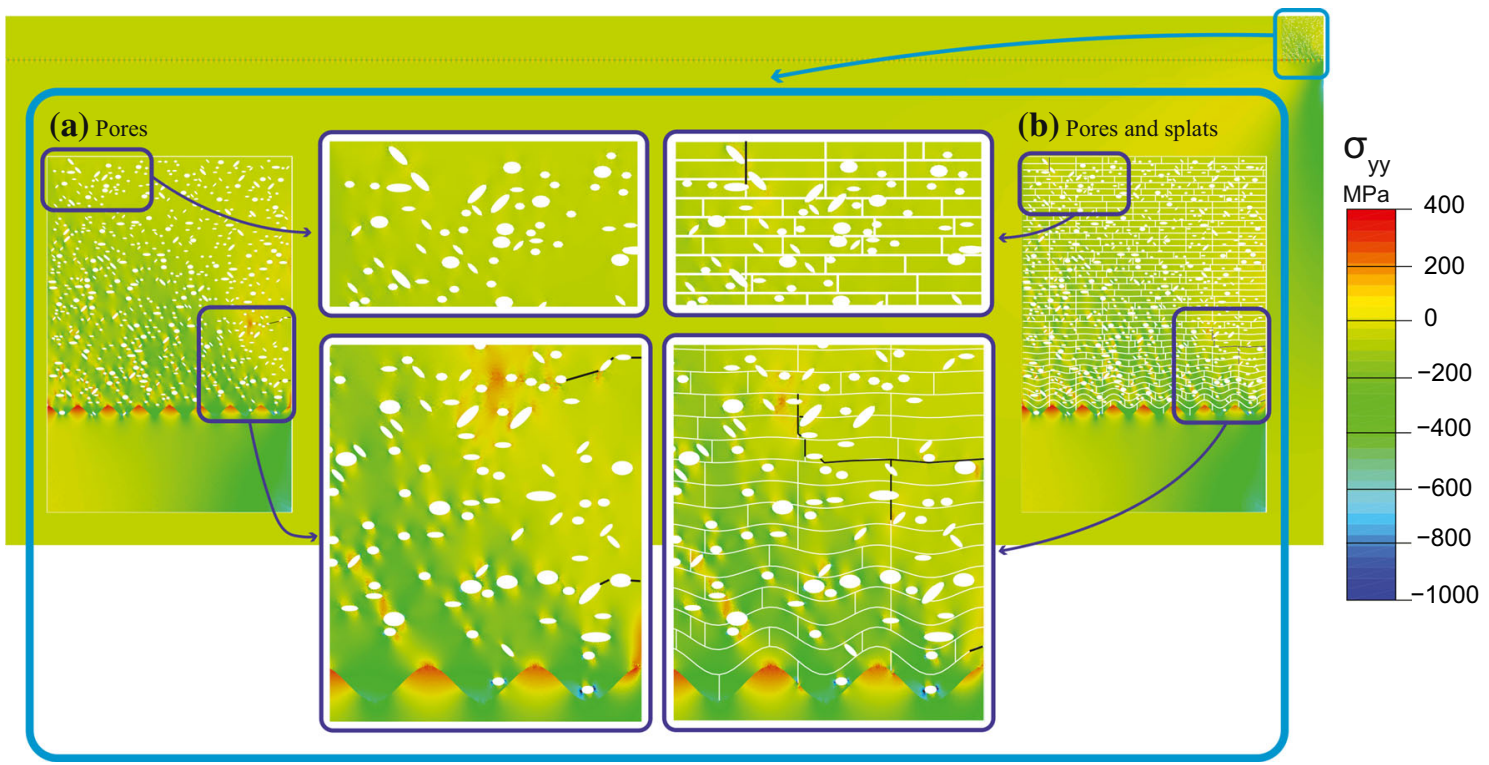

Fig. 3 Stress distribution $\left(\sigma_{y y}\right)$ and failure behavior of $\mathrm{TBC}$ at $T=30{ }^{\circ} \mathrm{C}$ for two distinct microstructural configurations. (a) Pores and (b) combined splats and pores

Table 3 Effect of TBC microstructural features

\begin{tabular}{lcc}
\hline Microstructural features & Crack initiation temperature, ${ }^{\circ} \mathrm{C}$ & Total crack length at room temperature, $\mathrm{mm}$ \\
\hline Dense (no defects) & 330 & 0.16 \\
Pores & $310 \pm 50$ & $0.07 \pm 0.01$ \\
Splat interfaces & $800 \pm 5$ & $0.28 \pm 0.03$ \\
Splat interfaces and pores & $680 \pm 16$ & $0.22 \pm 0.05$ \\
\hline
\end{tabular}

compared to the splat interfaces only case indicates an increased resistance against crack nucleation during cooling). In terms of the total crack length, the presence of pores has a positive effect for both the configurations (with and without splat interfaces). However, this effect is more pronounced for the microstructure without splat interfaces. For instance, the total crack length of the dense TBC is approximately two times higher than the TBC with pores. This is due to the fact that the presence of pores increases the TBC compliance which reduces the strain energy contribution to the crack driving force (Ref 32 ). The positive influence of the pores on TBC lifetime is reported in the literature through experimental measurements (Ref $33,34)$. On the other hand, a TC with splat interfaces leads to early crack initiation and also to a larger crack length, which is expected because of the weak interface. From Table 3, it can be concluded that the presence of pores results in an improved resistance of the TBC against fracture whereas the presence of splat interfaces is detrimental to the TBC. To understand the effect of splat interfaces on TBC failure behavior, parametric simulations
Table 4 Summary of splat interface and pore geometrical parameter used

\begin{tabular}{lc}
\hline Microstructural features & Geometrical parameters \\
\hline Splat interface waviness $(C)$ & $0,0.4,0.8,1$ \\
Splat interface spacing $(h)$ & $6.25,12.5,25(\mu \mathrm{m})$ \\
Porosity $\left(V_{\mathrm{f}}\right)$ & $0,10,15,20(\%)$ \\
\hline
\end{tabular}

are carried out in the next section for different geometries and fracture properties of the splat interfaces.

\section{Parametric Simulation}

The parametric studies are carried out for three different parameters such as splat interface waviness, spacing and porosity, and the variations are listed in Table 4. The simulation results are discussed in terms of crack initiation temperature and total crack length. Similar to the study on effect of TBC microstructural features, five different realizations are utilized for each parametric case to obtain the 
statistical variation due to the random distribution of pores and vertical splat interfaces.

\section{Effect of Splat Interface Waviness}

To investigate the effect of splat interface waviness, four different waviness values $0,0.4,0.8$ and 1 are analyzed. The splat interface spacing and porosity are fixed with a value of $12.5 \mu \mathrm{m}$ and $10 \%$, respectively. The waviness value of 0 results in flat interfaces in the computational domain, whereas the waviness of 1 corresponds to the cosine curve with specified amplitude of $10 \mu \mathrm{m}$ for all the splat interfaces (see Eq 1). For other waviness values, the amplitude of the splats (cosine curve) is scaled linearly with the increase in number of splat interfaces.

The results of the simulations are summarized in Fig. 4 to show the influence of splat waviness on initiation temperature and total crack length. From Fig. 4(a), it can be observed that the crack initiation temperature shows a weak dependency on splat interface waviness. This is due to the fact that the crack initiation is governed mostly by the free edge stress which is maximum at a region away from the $\mathrm{TC} / \mathrm{BC}$ interface. In this region, the change in splat interface amplitude for waviness values less than 1 is minimal because, with increase in number of splat interfaces, there is a decrease in amplitude. For a waviness value of 1 , the amplitude of the splat interfaces remain unaffected which results in a trend toward delay in crack initiation as shown in Fig. 4(a).

In terms of total crack length, the splat interface waviness shows a significant influence especially for the waviness of 0 and 1 as shown in Fig. 4(b). The crack length for the TBC with flat splat interfaces (waviness $=0$ ) is sensitive to the distribution of pores. Two out of five realizations show complete failure of the $\mathrm{TC}$ with major cracking close to the $\mathrm{TC} / \mathrm{BC}$ interface. Hence, the statistical variation of total crack length for zero waviness is considered as inconclusive. This behavior is due to the presence of a flat interface which favors the linking and propagation of microcracks. In addition, the formation of microcracks is influenced by the distribution of different pore features. Complete failure of the TC occurs if these microcracks reach a critical total length. For the splat interfaces with waviness of 0.4 and 0.8 , the total crack length remains almost the same and it decreases by a factor of 2 for the waviness of 1 . Thus, in general, it can be concluded that an increase in splat interface waviness improves the resistance against thermal fracture of the TBC. These findings are in agreement with indentation tests on APS TBC samples (Ref 21) that show that the fracture resistance is indeed improved significantly for TBCs with rough splat interfaces. Similarly, thermal cycling studies (Ref 35,36 ) on TBC also conclude that the bond coat roughness has a significant effect on TBC lifetime with higher roughness leading to an increase in TBC lifetime.

\section{Effect of Splat Interface Spacing}

Another parameter of interest is the splat interface spacing, which is a direct measure of the splat thickness. In the current TBC modeling setup, splat interface spacing affects both the interface waviness and the number of weak interfaces within the TC layer (refer Eq 1). Hence, the variations in splat interface spacing may have a significant effect on the TBC fracture characteristics. In order to study this effect, four different splat interface spacing given by $6.25,12.5,25$ and $50 \mu \mathrm{m}$ are considered. The values for splat interface waviness and volume fraction are kept constant at 0.8 and $10 \%$, respectively. The results obtained from the analysis are summarized in terms of crack initiation temperature and total crack length and are shown in Fig. 5. As observed in Fig. 5(a), the crack initiation temperature remains almost constant for the range of splat interface spacing considered. Nevertheless, for the reference configuration with only pores (no splats) given in Table 3, the crack initiation temperature is significantly lower than the initiation values reported in Fig. 5(a). In terms of total crack length, the results indicate that the total crack length decreases nonlinearly with the increase in
Fig. 4 Variation of (a) crack initiation temperature and (b) total crack length for different splat interface waviness of 0 (flat), $0.4,0.8$ and 1 with the fixed splat interface spacing of $12.5 \mu \mathrm{m}$ and volume fraction of $10 \%$. The total crack length for zero waviness (flat interface) is inconclusive since it does not include two fully failed samples out of five simulations

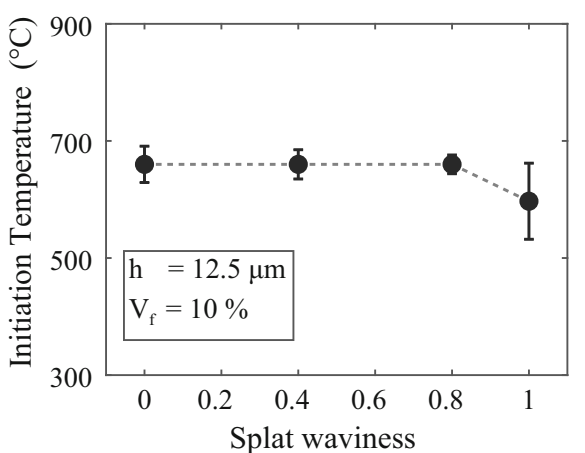

(a)

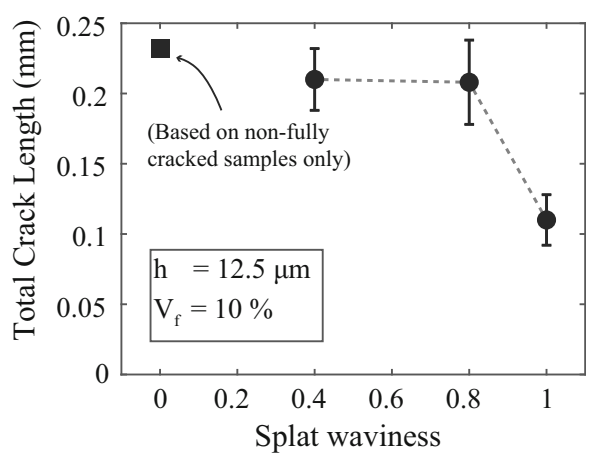

(b) 
Fig. 5 Variation of (a) crack initiation temperature and (b) total crack length for different splat interface spacing of 6.25 , $12.5,25$ and $50 \mu \mathrm{m}$ with the fixed splat interface waviness of 0 and volume fraction of $10 \%$

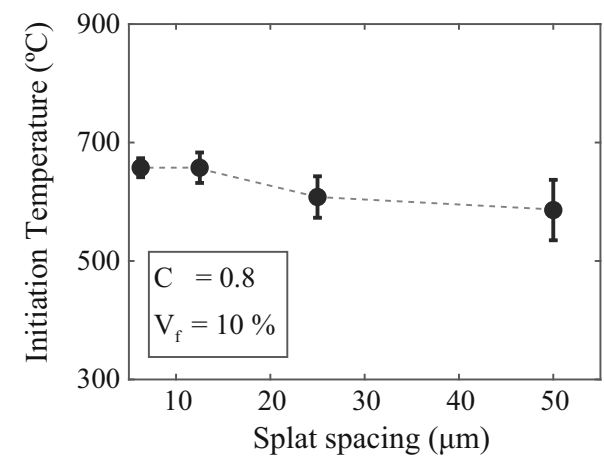

(a)

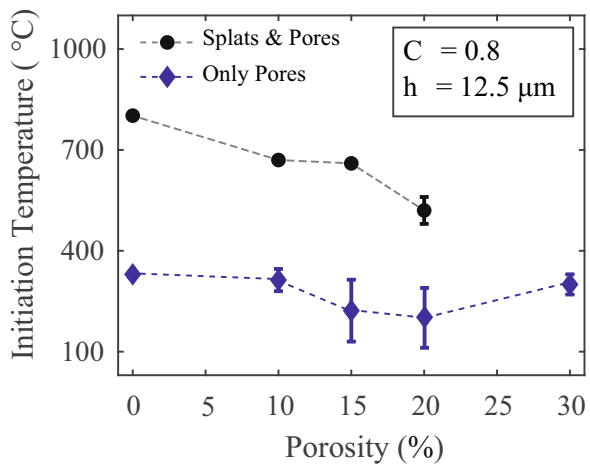

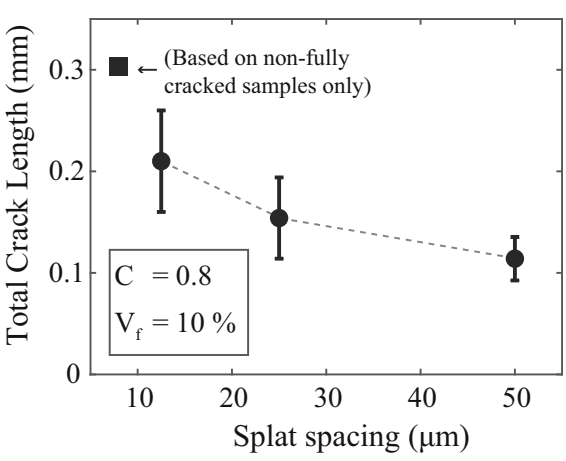

(b)

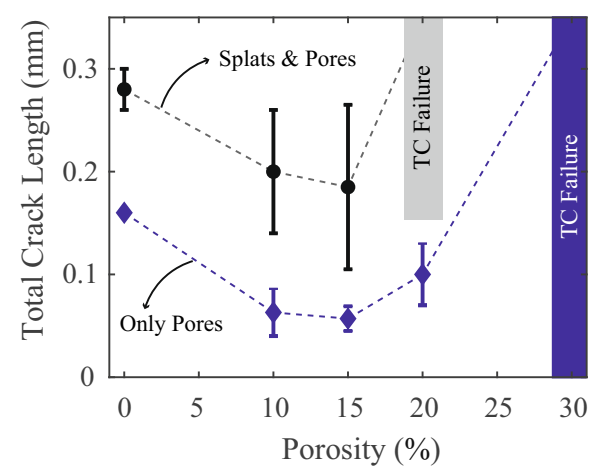

Fig. 6 Variation of (a) crack initiation temperature and (b) total crack length for different volume fractions of 0 (dense), $10,15,20,30 \%$ with the fixed splat interface waviness of 0 and splat interface spacing of 12.5 $\mu \mathrm{m}$ interfaces leads to early crack initiation when compared to the TC layer with only pores as shown in Fig. 6(a). The crack initiation temperature remains approximately constant for the TC layer with only pores, whereas the crack initiation temperature decreases nonlinearly with an increase in porosity for the microstructure with combined splat interfaces and pores. For the TC layer with combined splat interfaces and pores, the crack initiation temperature of $10 \%$ porosity decreases from 800 to $700{ }^{\circ} \mathrm{C}$ when compared with the zero porosity case. Upon further increase in porosity, the crack initiation temperature remains almost constant up to $V_{\mathrm{f}}=15 \%$ after which it decreases to $550{ }^{\circ} \mathrm{C}$ for $V_{\mathrm{f}}=20 \%$. In terms of total crack length, it can be seen that for both cases (with and without splat interfaces) the trend remains almost the same. The presence of pores improves the fracture resistance of the TC compared to the dense TBC (see Fig. 6b). This is due to the decrease in strain energy contribution to the crack driving force with porosity as explained in the section on the effect of TBC microstructural features. The scatter in total crack length values between individual simulations for a nominally identical sets of input parameters is attributed to the complex interaction of different pore features and also its interaction with the splat interface. Also, it is important to note that complete failure of TBC occurs for a critical value of the porosity is $\mathrm{V}_{f}=30 \%$ for TC with pores and $\mathrm{V}_{f}=20 \%$ for $\mathrm{TC}$ with combined splat 
interface and pores. The optimal porosity value remains constant at $\mathrm{V}_{f}=15 \%$ for both microstructural configurations.

\section{Effect of Fracture Properties}

As discussed earlier, the constitutive behavior of the splat interface is governed by its fracture parameters. Hence, the variation of these parameters significantly influences the fracture behavior of the TBC system. To study this effect, a parametric analysis is carried out for distinct material properties, expressed as ratios of a reference benchmark material with fracture strength $\sigma_{0}^{\mathrm{TC}}$ and fracture energy $G_{0}^{\mathrm{TC}}$, as follows:

$$
\begin{aligned}
\sigma^{\mathrm{TC}} & =f \sigma_{0}^{\mathrm{TC}}, \\
G^{\mathrm{TC}} & =f G_{0}^{\mathrm{TC}}
\end{aligned}
$$

where $f$ is the fracture ratio. Note that both properties are varied using the same fracture ratio $(f)$.

The parametric study is done with fixed splat interface waviness of 0.8 , splat interface spacing of $12.5 \mu \mathrm{m}$ and porosity of $10 \%$. Two distinct normal $\left(f_{\mathrm{n}}\right)$ and shear fracture $\left(f_{\mathrm{s}}\right)$ ratios are considered to analyze the influence of normal and shear fracture properties of the splat interfaces. For each fracture ratio, the simulations were carried out for five different values given by $0.5,0.75,1,1.5$ and 2 . While studying the influence of one fracture ratio, the other fracture ratio is fixed at the value of 1 . In other words, to study the effect of splat interface normal fracture properties, the normal fracture ratio $\left(f_{\mathrm{n}}\right)$ is varied with values given by $0.5,0.75,1,1.5$ and 2 for a fixed shear ratio $\left(f_{\mathrm{s}}\right)$ of 1 and vice versa.

Crack initiation temperature and total crack length for both normal and shear fracture ratios are shown in Fig. 7. From Fig. 7(a), it can be observed that the crack initiation temperature decreases almost linearly with increase in normal fracture ratio. For the variations in shear fracture ratio $\left(f_{\mathrm{s}}\right)$, the crack initiation temperature remains unaffected as shown in Fig. 7(c). This is because the normal fracture properties, being always less than the shear fracture properties for the splat interface, are the most critical values that control the crack initiation. In terms of crack length, the results indicate that the variation of the total crack length with the normal fracture ratio is not linear as shown in Fig. 7(b). In particular, a drastic increase in damage is observed in the TC layer when the normal fracture ratio is decreased to a value of 1 [i.e., total crack length increases abruptly by a factor of 2.5 when compared to the fracture ratio $\left(f_{\mathrm{n}}\right)$ of 1.5]. Similarly, the results shown in Fig. 7(d) are parameter value sensitive particularly, when the shear fracture ratio is decreased below a certain critical value. For instance, the complete failure of the TBC occurs when the shear fracture ratio is decreased below the value of 1 . The critical value for the complete TBC failure is decided by the value of the fracture parameters for which the crack coalescence is favoured.

\section{Effect of TGO Thickness}

The above reported simulations are carried out for the assprayed TBC system under single thermal cycle. However, during thermal cyclic operation, the oxidation of the bond coat results in growth of an additional layer called the thermally grown oxide layer (TGO). The growth of this additional layer affects both the stress distribution and the crack evolution pattern due to its strong CTE mismatch with the adjacent layers. Complete failure of the TBC occurs when the TGO thickness is in the range of 8 to 12 $\mu \mathrm{m}(\operatorname{Ref} 37,38)$. In this section, the influence of TGO on TBC fracture behavior is analyzed. Due to computational constraints, this effect is studied parametrically for a single cycle. This is based on the assumption that the TBC will undergo cracking only after the TGO thickness is increased to a specified value. Simulations are carried out for four different TGO thicknesses values given by $t_{\mathrm{TGO}}=0,3,6$ and $9 \mu \mathrm{m}$. The microstructural features of the TC layer such as porosity, splat interface waviness and spacing are kept constant with a value of $10 \%, 0.8$ and $12.5 \mu \mathrm{m}$, respectively. The pores are distributed randomly in the TC layer with pore characteristics specified in Table 1 .

The normal stress distribution in the vertical direction $\left(\sigma_{y y}\right)$ and the crack pattern in the TBC are shown in Fig. 8 for two different TGO thicknesses $t_{\mathrm{TGO}}=0 \mu \mathrm{m}$ (left inset) and $9 \mu \mathrm{m}$ (right inset). From Fig. 8, it can be observed that the presence of TGO significantly influences the stress distribution and cracking pattern in the TBC. The TGO thickness $t_{\mathrm{TGO}}=9 \mu \mathrm{m}$ leads to complete delamination failure of the TBC with majority of cracking on the splat interface close to the TC/TGO boundary, whereas for the TBC without a TGO layer $\left(t_{\mathrm{TGO}}=0 \mu \mathrm{m}\right)$ only limited cracking is observed. This is due to the altered TBC stress fields especially close to the TC/TGO interface. The stress concentration in the TBC is governed by two distinct parameters, one due to the presence of pores and the other due to the CTE mismatch between the layers for both cases (with and without TGO layer). However, for the TBC with TGO layer, the stress concentration close to the interface is amplified as shown in Fig. 8. This is attributed to the strong CTE mismatch between the TGO and the other layers (TC and $\mathrm{BC}$ ) compared to the TBC without TGO layer. Furthermore, the presence of TGO also influences the stress concentration in the vicinity of pores close to the TC/TGO interface. For instance, the vertical normal stress $\left(\sigma_{y y}\right)$ at the proximity of pores close to the TGO layer is largely 
Fig. 7 Variation of (a) and (c) crack initiation temperature and (b) and (d) total crack length of TBC with random representation of pores for different splat interface normal and shear strength values

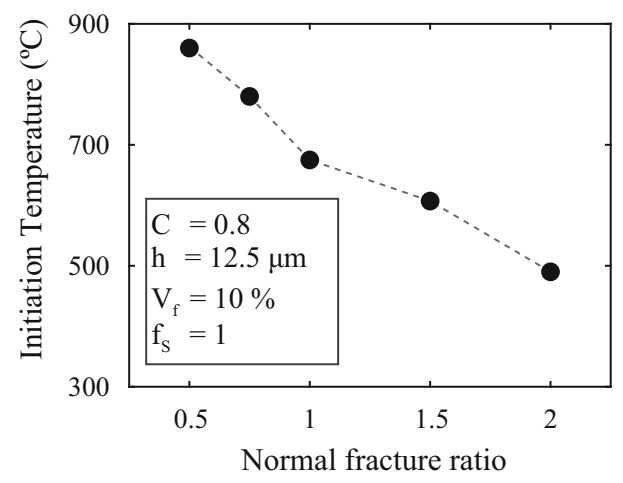

(a)

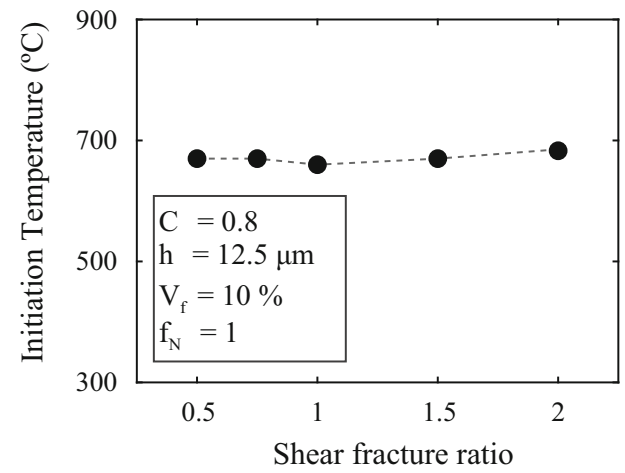

(c)

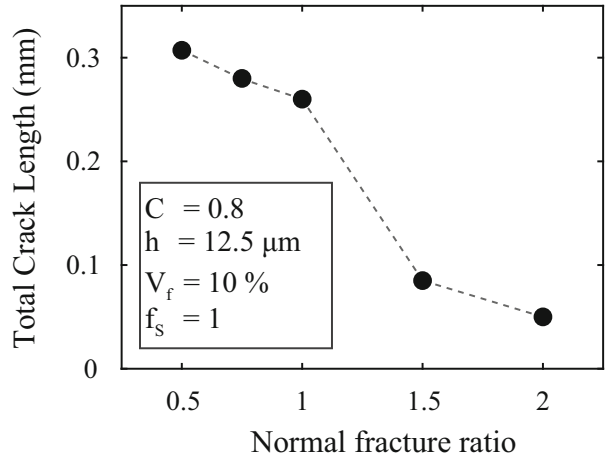

(b)

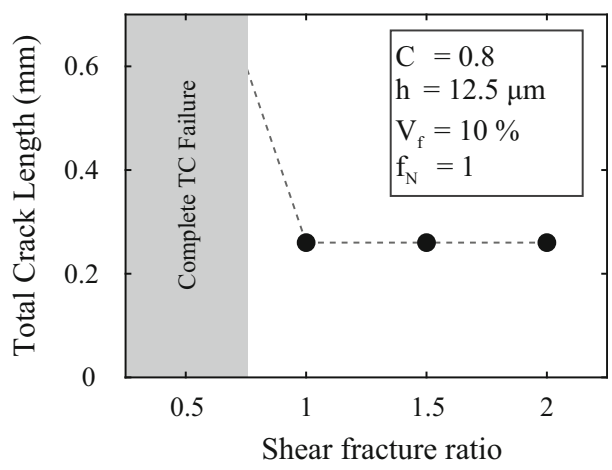

(d)

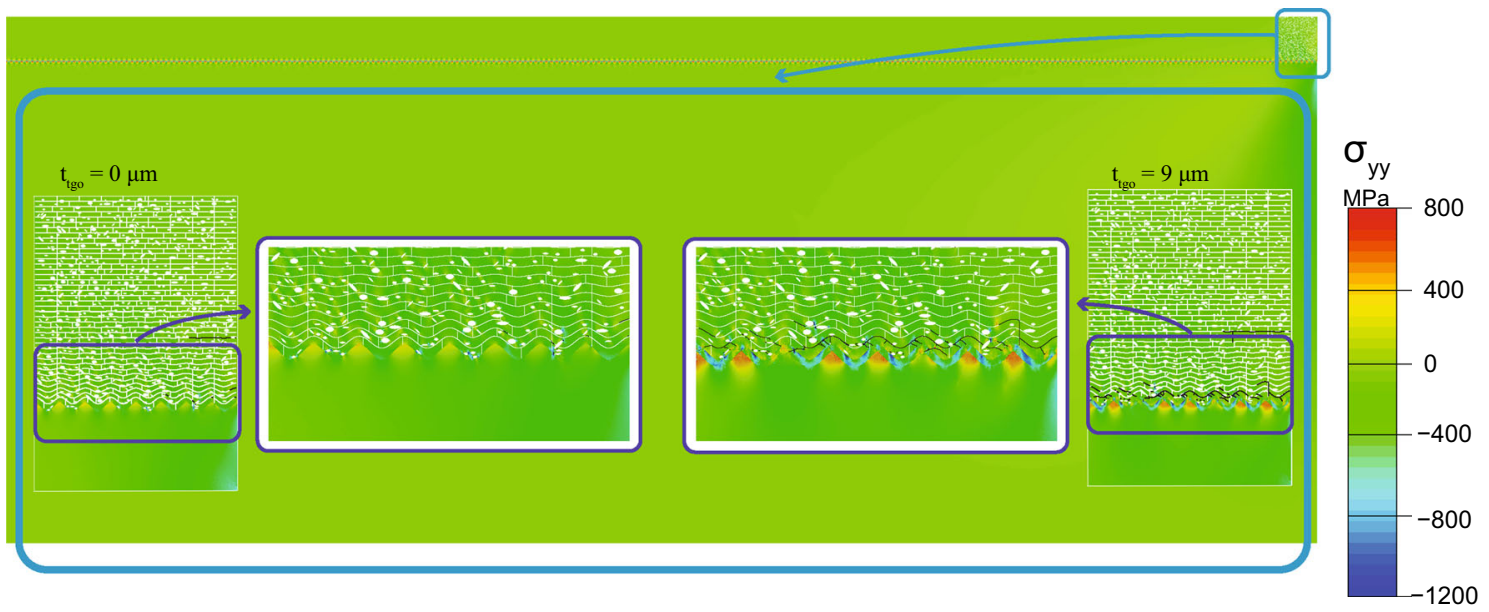

Fig. 8 Stress distribution in TBC with random microstructural pores at $T=30{ }^{\circ} \mathrm{C}$ for TGO thickness of 0 and $9 \mu \mathrm{m}$

tensile for TBC with TGO layer, whereas the stress fields are mostly compressive for TBC without TGO layer. The stress concentration close to the TC/TGO interface is further amplified with the TGO thickness. This results in early initiation and propagation of microcracks at the horizontal splat interface close to the TGO layer. Complete failure of the TBC occurs when these microcracks coalesce to form a major delamination crack. It is also important to note that the edge crack in the TC region is minimally influenced by the TGO layer when compared to the cracking in the TC region close to the TC/TGO interface. This is expected as the stress distribution in the TC region sufficiently away from the TC/TGO interface is less affected by the presence of the TGO layer. The influence of the TGO thickness on the TBC fracture performance is given in Fig. 9 in terms of the total crack length. The total crack length is plotted as a function of four considered TGO thicknesses. From Fig. 9, it can be seen that the increase in TGO thickness decreases the mechanical integrity of the TBC system. A TGO thickness of $9 \mu \mathrm{m}$ results in complete failure. This behavior 


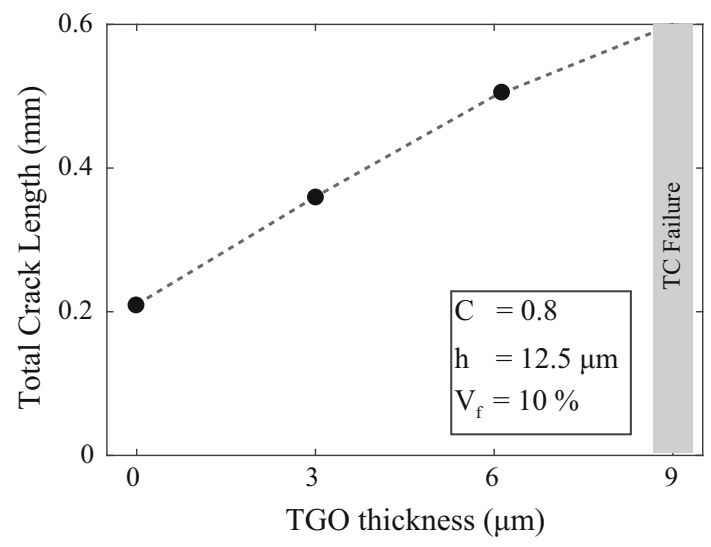

Fig. 9 Variation of total crack length for different TGO thickness 0, 3,6 and $9 \mu \mathrm{m}$

is due to the increase in stress concentration with TGO layer thickness as discussed above.

\section{Conclusions}

Thermal fracture behavior of the APS TBC with explicitly modeled splat interface and pores is analyzed using a cohesive elements based finite element method. The influence of splat interface geometric characteristics (such as the roughness of the planes in which the splat interfaces are located and their spacing), porosity and splat interface material parameter (fracture properties) on TBC fracture behavior is studied through parametric simulations. The results of the parametric studies are reported in terms of the crack initiation temperature and total microcrack length in the computational domain upon cooling down from the peak temperature of $1100{ }^{\circ} \mathrm{C}$ to room temperature $\left(30{ }^{\circ} \mathrm{C}\right)$, whereby both the splat interface and pores are found to exhibit a significant influence on the fracture behavior. The following conclusions are drawn from the parametric finite element investigations.

1. Microstructural features of the TBCs play a significant role in determining its failure characteristics. With reference to the fully dense TBC system, the presence of pores improves the fracture resistance whereas the presence of splat interface leads to early and more extensive failure of the TBC.

2. The influence of splat interface waviness on the crack initiation temperature is limited. The crack length for the flat splat interface is sensitive to the distribution of pores. In general, the increase in splat interface waviness improves the TBC fracture resistance.

3. Splat interface spacing did not have a significant influence on the initiation temperature, whereas the total crack length decreases with an increase in splat interface spacing.
4. The presence of pores improves the resistance against TBC fracture for both microstructural configurations considered. However, porosity beyond a critical value leads to early failure.

5. On the effect of splat interface fracture properties, the crack initiation temperature scales more or less linearly with normal tensile strength of the splat interfaces. In terms of crack length, the results are sensitive when the fracture ratio values are reduced to a value below a critical level. Complete failure of TBC occurs when shear fracture ratio is decreased below a value of 1 .

6. The increase in TGO thickness proportionally decreases the mechanical integrity of the TBC system.

The results and insights as reported here aim to clarify understanding the effect of TBC microstructure especially splat interfaces and pores on failure behavior, which in turn can aid in optimizing the processing and spraying parameters for improved performance/lifetime. Based on the current simulations, TBC systems with an optimal porosity value, having the splat interfaces arranged in non-planar configuration, having a large splat interface spacing and a high splat interface tensile strength perpendicular to the splat interface plane, should give the best thermal cracking resistance.

Acknowledgments This work was funded in part by the European Union's seventh framework program (FP7) through the NMP SAMBA project (Grant Number 309849). We extend our sincere thanks to our collaborator and SAMBA program leader Prof. W.G. Sloof for his valuable support and interactive discussions. We also acknowledge the use of a Matlab code to generate splat interfaces microstructures as developed in a companion project (Ref 39$)$.

Open Access This article is distributed under the terms of the Creative Commons Attribution 4.0 International License (http://crea tivecommons.org/licenses/by/4.0/), which permits unrestricted use, distribution, and reproduction in any medium, provided you give appropriate credit to the original author(s) and the source, provide a link to the Creative Commons license, and indicate if changes were made.

\section{References}

1. A. Feuerstein, J. Knapp, T. Taylor, A. Ashary, A. Bolcavage, and N. Hitchman, Technical and Economical Aspects of Current Thermal Barrier Coating Systems for Gas Turbine Engines by Thermal Spray and EBPVD: A Review, J. Therm. Spray Technol., 2008, 17(2), p 199-213

2. S. Gong and Q. Wu, Processing, Microstructures and Properties of Thermal Barrier Coatings by Electron Beam Physical Vapor Deposition (EB-PVD), Woodhead Publishing, In Therm. Barrier Coatings, 2011, p 115-131

3. J.L. Xu and K.A. Khor, Plasma Spraying for Thermal Barrier Coatings: Processes and Applications, in Thermal Barrier Coating. (Woodhead Publishing, 2011), pp. 99-114

4. A. Abdul-Aziz, Durability Modeling Review of Thermal- and Environmental-Barrier-Coated Fiber-Reinforced Ceramic Matrix Composites Part I, Materials, 2018, 11(7), p 1251-1267 
5. D.E. Wroblewski, R. Khare, and M. Gevelber, Solidification Modeling of Plasma Sprayed TBC: Analysis of Remelt and Multiple Length Scales of Rough Substrates, J. Therm. Spray Technol., 2002, 11(2), p 266-275

6. G.R. Li, G.J. Yang, C.X. Li, and C.J. Li, Sintering Characteristics of Plasma-Sprayed TBCs: Experimental Analysis and an Overall Modelling, Ceram. Int., 2018, 44(3), p 2982-2990

7. C. Lamuta, G. Di Girolamo, and L. Pagnotta, Microstructural, Mechanical and Tribological Properties of Nanostructured YSZ Coatings Produced with Different APS Process Parameters, Ceram. Int., 2015, 41(7), p 8904-8914

8. E. Lugscheider and R. Nickel, Finite Element Simulation of a Coating Formation on a Turbine Blade During Plasma Spraying, Surf. Coatings Technol., 2003, 174, p 475-481

9. M. Mutter, G. Mauer, R. Mücke, O. Guillon, and R. Vaßen, Correlation of Splat Morphologies with Porosity and Residual Stress in Plasma-Sprayed YSZ Coatings, Surf. Coatings Technol., 2017, 318, p 157-169

10. Z. Wang, A. Kulkarni, S. Deshpande, T. Nakamura, and H. Herman, Effects of Pores and Interfaces on Effective Properties of Plasma Sprayed Zirconia Coatings, Acta Mater., 2003, 51(18), p 5319-5334

11. J. Malzbender and R.W. Steinbrech, Fracture Resistance of Atmospheric Plasma Sprayed Thermal Barrier Coatings, Surf. Coatings Technol., 2012, 209, p 97-102

12. T. Wakui, J. Malzbender, and R.W. Steinbrech, Strain Analysis of Plasma Sprayed Thermal Barrier Coatings Under Mechanical Stress, J. Therm. Spray Technol., 2004, 13(3), p 390-395

13. G.R. Li, G.J. Yang, X.F. Chen, C.X. Li, and C.J. Li, Strain/ Sintering Co-induced Multiscale Structural Changes in PlasmaSprayed Thermal Barrier Coatings, Ceram. Int., 2018, 44(12), p 14408-14416

14. A. Kulkarni, Z. Wang, T. Nakamura, S. Sampath, A. Goland, H. Herman, J. Allen, J. Ilavsky, G. Long, J. Frahm, and R.W. Steinbrech, Comprehensive Microstructural Characterization and Predictive Property Modeling of Plasma-Sprayed Zirconia Coatings, Acta Mater., 2003, 51(9), p 2457-2475

15. P. Michlik and C. Berndt, Image-Based Extended Finite Element Modeling of Thermal Barrier Coatings, Surf. Coatings Technol., 2006, 201(6), p 2369-2380

16. L. Lu, F.C. Wang, Z. Ma, and Q.B. Fan, Anisotropic Effect of Splat Interface on Thermal Conductivity of Plasma Sprayed YSZ Coating, Surf. Coatings Technol., 2013, 235, p 596-602

17. X. Guo, W. Zhao, Y. Zeng, C. Lin, and J. Zhang, Effects of Splat Interfaces, Monoclinic Phase and Grain Boundaries on the Thermal Conductivity of Plasma Sprayed Yttria-Stabilized Zirconia Coatings, Coatings, 2019, 9(1), p 26

18. J. Krishnasamy, S.A. Ponnusami, S. Turteltaub, and S. van der Zwaag, Computational Investigation of Porosity Effects on Fracture Behavior of Thermal Barrier Coatings, Ceram. Int., 2019, 45(16), p 20518-20527

19. M. Białas, Finite Element Analysis of Stress Distribution in Thermal Barrier Coatings, Surf. Coatings Technol., 2008, 202(24), p 6002-6010

20. Z.Y. Wei and H.N. Cai, Stress States and Crack Behavior in Plasma Sprayed TBCs Based on a Novel Lamellar Structure Model with Real Interface Morphology, Ceram. Int., 2019, 45(14), p 16948-16962

21. J. Huang, W. Wang, X. Lu, S. Liu, and C. Li, Influence of Lamellar Interface Morphology on Cracking Resistance of Plasma-Sprayed YSZ Coatings, Coatings, 2018, 8(5), p 187

22. C. Li, X. Zhang, Y. Chen, J. Carr, S. Jacques, J. Behnsen, M. di Michiel, P. Xiao, and R. Cernik, Understanding the Residual Stress Distribution Through the Thickness of Atmosphere Plasma Sprayed (APS) Thermal Barrier Coatings (TBCs) by High Energy
Synchrotron XRD; Digital Image Correlation (DIC) and Image Based Modelling, Acta Mater., 2017, 132, p 1-12

23. W.G. Sloof. Self-Healing Thermal Barrier Coatings for Prolonged Lifetime (Funded by EU-FP7, Grant Number 309849)

24. J. Krishnasamy, S.A. Ponnusami, S. Turteltaub, and S. van der Zwaag, Modelling the Fracture Behaviour of Thermal Barrier Coatings Containing Healing Particles, Mater. Des., 2018, 157, p 75-86

25. S.A. Ponnusami, S. Turteltaub, and S. van der Zwaag, CohesiveZone Modelling of Crack Nucleation and Propagation in Particulate Composites, Eng. Fract. Mech., 2015, 149, p 170-190

26. Y. Wu, P. Hsu, Y. Wang, M.H. McCay, D.E. Croy, D. Moreno, L. He, C. Wang, and H. Zhang, Laser Thermal Gradient Testing and Fracture Mechanics Study of a Thermal Barrier Coating, J. Therm. Spray Technol., 2019, 28(6), p 1239-1251

27. A. Hillerborg, M. Modéer, and P.E. Petersson, Analysis of Crack Formation and Crack Growth in Concrete by Means of Fracture Mechanics and Finite Elements, Cem. Concr. Res., 1976, 6(6), p 773-781

28. S.R. Choi and N.P. Bansal, Mechanical Behavior of Zirconia/ Alumina Composites, Ceram. Int., 2005, 31(1), p 39-46

29. M. Munro, Evaluated Material Properties for a Sintered AlphaAlumina, J. Am. Ceram. Soc., 2005, 80(8), p 1919-1928

30. T.S. Hille, S. Turteltaub, and A.S.J. Suiker, Oxide Growth and Damage Evolution in Thermal Barrier Coatings, Eng. Fract. Mech., 2011, 78(10), p 2139-2152

31. R. Vaßen, G. Kerkhoff, and D. Stöver, Development of a Micromechanical Life Prediction Model for Plasma Sprayed Thermal Barrier Coatings, Mater. Sci. Eng. A, 2001, 303(1-2), p 100-109

32. M. Karger, R. Vaßen, and D. Stöver, Atmospheric Plasma Sprayed Thermal Barrier Coatings with High Segmentation Crack Densities: Spraying Process, Microstructure and Thermal Cycling Behavior, Surf. Coatings Technol., 2011, 206(1), p 16-23

33. M. Ahrens, R. Vaßen, D. Stöver, and S. Lampenscherf, Sintering and Creep Processes in Plasma-Sprayed Thermal Barrier Coatings, J. Therm. Spray Technol., 2004, 13(3), p $432-442$

34. N. Curry, N. Markocsan, L. Östergren, X.-H. Li, and M. Dorfman, Evaluation of the Lifetime and Thermal Conductivity of Dysprosia-Stabilized Thermal Barrier Coating Systems, J. Therm. Spray Technol., 2011, 22(6), p 864-872

35. W. Nowak, D. Naumenko, G. Mor, F. Mor, D.E. Mack, R. Vaßen, L. Singheiser, and W.J. Quadakkers, Effect of Processing Parameters on MCrAlY Bondcoat Roughness and Lifetime of APS-TBC Systems, Surf. Coatings Technol., 2014, 260, p 82-89

36. R. Eriksson, S. Sjöström, H. Brodin, S. Johansson, L. Östergren, and X.-H. Li, TBC Bond Coat-Top Coat Interface Roughness: Influence on Fatigue Life and Modelling Aspects, Surf. Coatings Technol., 2013, 236, p 230-238

37. H. Dong, G.J. Yang, C.X. Li, X.T. Luo, and C.J. Li, Effect of TGO Thickness on Thermal Cyclic Lifetime and Failure Mode of Plasma-Sprayed TBCs, J. Am. Ceram. Soc., 2014, 97(4), p 12261232

38. O. Trunova, T. Beck, R. Herzog, R.W. Steinbrech, and L. Singheiser, Damage Mechanisms and Lifetime Behavior of Plasma Sprayed Thermal Barrier Coating Systems for Gas TurbinesPart I: Experiments, Surf. Coatings Technol., 2008, 202(20), p 5027-5032

39. S. Murali, Micromechanical Modelling of Fracture Behaviour in Self-Healing Thermal Barrier Coatings. Master's Thesis, TUDelft, The Netherlands, 2017.

Publisher's Note Springer Nature remains neutral with regard to jurisdictional claims in published maps and institutional affiliations. 